PROCEEDINGS OF THE

AMERICAN MATHEMATICAL SOCIETY

Volume 138, Number 5, May 2010, Pages 1793-1801

S 0002-9939(10)10202-0

Article electronically published on January 7, 2010

\title{
UNIQUENESS AND INSTABILITY OF SUBSONIC-SONIC POTENTIAL FLOW IN A CONVERGENT APPROXIMATE NOZZLE
}

\author{
PAN LIU AND HAIRONG YUAN \\ (Communicated by Walter Craig)
}

\begin{abstract}
We proved uniqueness and instability of the symmetric subsonicsonic flow solution of the compressible potential flow equation in a surface with convergent areas of cross-sections. Such a surface may be regarded as an approximation of a two-dimensional convergent nozzle in aerodynamics. Mathematically these are uniqueness and nonexistence results of a nonlinear degenerate elliptic equation with Bernoulli type boundary conditions. The proof depends on maximum principles and a generalized Hopf boundary point lemma which was proved in the paper.
\end{abstract}

\section{INTRODUCTION}

This paper is a continuation of our study on transonic flows in nozzles 17, 18. A fundamental difficulty to this problem is the lack of special solutions in a physical nozzle. However, as motivated by 14, it is rather easy to construct various physically interesting special solutions, such as those (supersonic-subsonic) transonic shocks and (subsonic-supersonic) transonic flows observed in physical nozzles [4], for the steady compressible Euler equations in certain Riemannian manifolds [17, 18]. Therefore these manifolds may be regarded as approximate nozzles, and the study of the special flows in them would be of certain importance for the understanding of flows in physical nozzles. In the previous works [3, 10, 11, we and our collaborators have studied stability and uniqueness of transonic shocks for the potential flow equation or the complete Euler system, which involve free boundary problems of uniformly elliptic equations (for the potential flow equation) or an elliptic-hyperbolic composite system (for the Euler system). In [17, 18, we construct a special transonic flow solution and show its stability for the potential flow equation under perturbations of the potential function at the entry, which is a small perturbation result on nonlinear equations of mixed type. The present paper

Received by the editors April 27, 2009, and, in revised form, September 14, 2009.

2010 Mathematics Subject Classification. Primary 35J70, 35B50; Secondary 76H05.

The first author was supported in part by the National Science Foundation of China under Grants No. 10601017 and 10871126.

The second author (corresponding author) was supported in part by the National Science Foundation of China under Grant No. 10901052, Shanghai Chenguang Program (09CG20), a Special Research Fund for Selecting Excellent Young Teachers of the Universities in Shanghai sponsored by the Shanghai Municipal Education Commission, and the National Science Foundation (USA) under Grant DMS-0720925. 
is devoted to the uniqueness and instability of subsonic-sonic flow in a convergent approximate nozzle in the class of all $C^{2}$ functions satisfying certain reasonable restrictions. These are global results on degenerate elliptic equations. We first formulate the problem.

Let $\mathbf{S}^{1}$ be the standard unit circle in $\mathbf{R}^{2}$, and let $\mathcal{M}$ be the Riemannian manifold $\left\{(x, y) \in(0,1) \times \mathbf{S}^{1}\right\}$ with a metric $G=d x \otimes d x+n(x)^{2} d y \otimes d y$. Here $n(t)$ is a positive smooth function on $[0,1]$ and satisfies:

$$
\text { (i) } n^{\prime \prime}(t)>0 \text {; (ii) } n^{\prime}(t)<0 \text { for } t \in(0,1), n^{\prime}(1)=0 \text {. }
$$

Without loss of generality, we also assume that $n(1)=1$. We call $\mathcal{M}$ a convergent approximate nozzle. In the following, we take $(x, y) \in M=(0,1) \times(0,2 \pi)$ as a coordinate system in $\mathcal{M}$. Note that on $y=0$ and $y=2 \pi$ periodic conditions should be posed.

The potential flow equation governing steady irrotational isentropic perfect gas flows in $\mathcal{M}$ is (cf. [18])

$$
\begin{gathered}
n(x)^{2}\left(c^{2}-\left(\partial_{1} \varphi\right)^{2}\right) \partial_{11} \varphi-2 \partial_{1} \varphi \partial_{2} \varphi \partial_{12} \varphi+\left(c^{2}-\frac{1}{n(x)^{2}}\left(\partial_{2} \varphi\right)^{2}\right) \partial_{22} \varphi \\
+n(x) n^{\prime}(x)\left(c^{2}+\frac{1}{n(x)^{2}}\left(\partial_{2} \varphi\right)^{2}\right) \partial_{1} \varphi=0 .
\end{gathered}
$$

(Here and hereafter we set $\partial_{1}=\partial_{x}, \partial_{2}=\partial_{y}$.) A direct computation yields that this equation is of elliptic type if the flow is subsonic $\left(c^{2}>\left(\partial_{1} \varphi\right)^{2}+\left(\partial_{2} \varphi\right)^{2} / n(x)^{2}\right)$, and degenerate if the flow is sonic $\left(c^{2}=\left(\partial_{1} \varphi\right)^{2}+\left(\partial_{2} \varphi\right)^{2} / n(x)^{2}\right)$. We may compute the speed of sound $c$ by Bernoulli's law

$$
\frac{c^{2}}{\gamma-1}+\frac{\left(\partial_{1} \varphi\right)^{2}+\left(\partial_{2} \varphi\right)^{2} / n(x)^{2}}{2}=\frac{c_{0}}{\gamma-1},
$$

with $\gamma>1$ and $c_{0}>0$ two given constants. Let $p, \rho$ be the pressure and density of the flow, respectively. We use $p=\rho^{\gamma}$ as the state function and then $c=\sqrt{\gamma \rho^{\gamma-1}}$.

We call $\Sigma_{k}=\{k\} \times \mathbf{S}^{1}, k=0,1$, respectively the entry and the exit of the approximate nozzle $\mathcal{M}$. Obviously $\partial \mathcal{M}=\Sigma_{0} \cup \Sigma_{1}$. In the following we assume that

$$
\partial_{1} \varphi \geq 0 \text { on both } \Sigma_{0} \text { and } \Sigma_{1} \text {, }
$$

which means exactly that the gas flows into $\mathcal{M}$ on $\Sigma_{0}$ and flows out of $\mathcal{M}$ on $\Sigma_{1}$.

We study subsonic-sonic flows in $\mathcal{M}$. The boundary conditions are

$$
\begin{array}{cc}
\left(\partial_{1} \varphi\right)^{2}+\frac{\left(\partial_{2} \varphi\right)^{2}}{n(0)^{2}}=b_{0}^{2} & \text { on } \Sigma_{0}, \\
\left(\partial_{1} \varphi\right)^{2}+\left(\partial_{2} \varphi\right)^{2}=b_{1}^{2}:=\frac{2 c_{0}}{\gamma+1} & \text { on } \Sigma_{1},
\end{array}
$$

where $b_{0}, b_{1}$ are positive constants. Note that (1.3) and (1.4) are Bernoulli type conditions and the choice of $b_{1}$ shows that the flow is sonic at the exit. We choose $b_{0}$ to satisfy the algebraic equation

$$
b_{0}^{\gamma-1}\left(c_{0}-\frac{\gamma-1}{2} b_{0}^{2}\right) n(0)^{\gamma-1}=b_{1}^{\gamma+1}
$$

and $b_{0}<b_{1}$ so that the flow is subsonic at the entry. It is straightforward to show that there exists such a unique $b_{0}$. We pose these nonlinear Bernoulli conditions rather than Dirichlet or Neumann conditions, since from the physical point of view, prescribing density (pressure) at the entry and exit are more reasonable [3, 4. By Bernoulli's law (1.2), this consideration leads to (1.3) and (1.4). 
Now suppose the flow is symmetric, that is, depends only on $x$. Then (1.1) (1.4) are reduced to this problem of $u(x)=\varphi^{\prime}(x)$ :

$$
\begin{array}{cl}
\partial_{x}(n \rho u)=0, & x \in[0,1], \\
\frac{\gamma \rho^{\gamma-1}}{\gamma-1}+\frac{u^{2}}{2}=\frac{c_{0}}{\gamma-1}, & x \in[0,1], \\
u=b_{0}, & x=0, \\
u=b_{1}, & x=1 .
\end{array}
$$

Under the above choice of $b_{0}$, the velocity $u$ can be easily solved and the obtained flow is subsonic in $\mathcal{M} \cup \Sigma_{0}$, sonic on $\Sigma_{1}$, and always accelerates in $\mathcal{M}$ (i.e., $u^{\prime}(x)=$ $\left.\varphi^{\prime \prime}(x)>0\right)$. Therefore we may choose a potential function $\varphi_{b}=\varphi_{b}(x)$ with $\varphi_{b}^{\prime}=u$ being a special solution to problems (1.1)-(1.4).

Apart from stability, the question of whether this special solution is unique in the large also naturally arises, that is, under suitable assumptions, whether any solution to (1.1)-(1.4), modulo a constant, must be the $\varphi_{b}$ obtained above. The following results provide a positive answer to the uniqueness, but a negative one to the stability.

Theorem 1.1. (i) The only $C^{2}(\overline{\mathcal{M}})$ solution to problems (1.1) -(1.4) which satisfies $\left(\mathrm{H}_{2}\right)$ and

$$
\partial_{11} \varphi>0 \text { on } \Sigma_{1}
$$

is the symmetric solution $\varphi_{b}$ modulo a constant.

(ii) Under the same assumptions as in (i), but replacing (1.3) by

$$
\left(\partial_{1} \varphi\right)^{2}+\frac{\left(\partial_{2} \varphi\right)^{2}}{n(0)^{2}}=B(y) \text { on } \Sigma_{0},
$$

with $B(y)$ a positive function not identical to $b_{0}^{2}$ and satisfying either $B(y) \geq b_{0}^{2}$ or $B(y) \leq b_{0}^{2}$, then there will be no $C^{2}$ solution to the problems (1.1), (1.2), (1.11) and (1.4).

We remark that there are no extra requirements such as the flow to be subsonic in $\mathcal{M}$ to show the uniqueness. The only assumption (1.10) means that the flow is accelerating at the exit. From the study of transonic flows [9] and the nonuniqueness results claimed by many authors based on numerical simulations (cf. [15]) it seems that this assumption is necessary. Assertion (ii) shows in particular that the subsonic-sonic flow is not stable under perturbations of the density (or pressure, or speed) of the gas at the entry, provided the flow is accelerating at the exit, where it is sonic.

The rest of this paper, Section 2, is devoted to the proof of Theorem 1.1. The key point is to prove a version of the Hopf boundary point lemma applicable to points on characteristic degenerate boundaries for degenerate elliptic equations, which is Theorem 2.1 stated in Section 2.1. Although there is much impressive progress in the study of degenerate elliptic equations and mixed type equations in gas dynamics and other fields in these years (see [1, 2, 6, 17, 8, 9, 12, 13, 19, and the references therein), this generalized Hopf lemma seems to be new. It captures the remarkable property that for subsonic-sonic flow or transonic flow, the potential flow equation behaves like the heat equation $-\partial_{1} \varphi+\partial_{22} \varphi=0$ near the sonic line (the line where the equation is degenerate), and hence the lower order term $-\partial_{1} \varphi$ is essential in studying these degenerate elliptic or mixed type equations (cf. [2, 18]). 
This observation, obtained by studying special subsonic-sonic flows and transonic flows in an approximate nozzle, would be important for studying the flows in a physical nozzle.

\section{Proof OF MAIN THEOREMS}

2.1. A generalized Hopf lemma. We first present a version of the Hopf lemma applicable to degenerate elliptic operators and characteristic boundary points, which may be regarded as a generalization of results such as Lemma 3.4 in [5] or Lemma 7.1.7 in [16] and is of independent interest.

Let $D$ be a bounded domain of class $C^{2}$ in $\mathbb{R}^{n}$ with boundary $\partial D$, and let

$$
L:=\sum_{i, j=1}^{n} a^{i j}(x) \partial_{i j}+\sum_{i=1}^{n} b^{i}(x) \partial_{i}+c(x)
$$

be a second order degenerate elliptic operator in $D$ (that is, $a^{i j}(x) \xi_{i} \xi_{j} \geq 0$ for all $\xi=\left(\xi_{1}, \cdots, \xi_{n}\right) \in \mathbb{R}^{n}$ and $\left.x \in D\right)$. In what follows we assume that $a^{i j}(x), b^{i}(x)$, $c(x)$ are all bounded and continuous functions in $D$. Let $P$ be a point on $\partial D$ and assume, in a neighborhood $U \subset \mathbb{R}^{n}$ of $P$, that the boundary $\partial D$ has the form $\left\{x=\left(x_{1}, \cdots, x_{n}\right) \in \mathbb{R}^{n}: x_{n}-f\left(x_{1}, \cdots, x_{n-1}\right)=0\right\}$ with $f$ a $C^{2}$ function, and

$U \cap D$ lies in $\left\{x_{n}>f\left(x_{1}, \cdots, x_{n}\right)\right\}$. Then $\nu=\left(-\frac{\partial f}{\partial x_{1}}, \cdots,-\frac{\partial f}{\partial x_{n-1}}, 1\right)$ is an interior normal vector to $\partial D$. We have the following result.

Theorem 2.1. Let $u$ be a $C^{2}(D) \cap C^{1}(\bar{D})$ function that attains a strict minimum (maximum) at $P \in \partial D$. Also assume that in a neighborhood $U \cap \bar{D}$ of $P$ we have $c=0$ and

$$
-\sum_{i=1}^{n-1} b^{i} \frac{\partial f}{\partial x_{i}}+b^{n}-\sum_{i, j=1}^{n-1} a^{i j} \frac{\partial^{2} f}{\partial x_{i} \partial x_{j}}>0 \quad \text { at } P .
$$

Then if

$$
L u \leq 0 \quad(\geq 0) \quad \text { in } D
$$

we have

$$
\frac{\partial u}{\partial \nu}>0 \quad(<0) \quad \text { at } P .
$$

If $c \leq 0$, the same conclusion holds provided that $u(P) \leq 0(\geq 0)$.

Proof. First note that we may choose the following transformation,

$$
y_{1}=x_{1}-x_{1}(P), y_{2}=x_{2}-x_{2}(P), \cdots, y_{n}=x_{n}-f\left(x_{1}, \cdots, x_{n-1}\right),
$$

to map $P$ to $O$ in $y$-coordinates and straighten $\partial D$ locally to $y_{n}=0$ near the origin $O$. Denote the image of $U$ to be $V$. 
It follows that the operator $L$ can be written in the form

$$
\begin{aligned}
L= & \sum_{i, j=1}^{n} a^{i j}(x) \frac{\partial^{2}}{\partial x_{i} \partial x_{j}}+\sum_{i=1}^{n} b^{i}(x) \frac{\partial}{\partial x_{i}}+c \\
= & \sum_{k, l=1}^{n}\left(\sum_{i, j=1}^{n} a^{i j} \frac{\partial y_{k}}{\partial x_{i}} \frac{\partial y_{l}}{\partial x_{j}}\right) \frac{\partial^{2}}{\partial y_{k} \partial y_{l}} \\
& +\sum_{l=1}^{n}\left(\sum_{i=1}^{n} b^{i} \frac{\partial y_{l}}{\partial x_{i}}+\sum_{i, j=1}^{n} a^{i j} \frac{\partial^{2} y_{l}}{\partial x_{i} \partial x_{j}}\right) \frac{\partial}{\partial y_{l}}+c \\
= & \sum_{k, l=1}^{n} \alpha^{k l}(y) \frac{\partial^{2}}{\partial y_{k} \partial y_{l}}+\sum_{l=1}^{n} \beta^{l}(y) \frac{\partial}{\partial y_{l}}+c,
\end{aligned}
$$

where $\alpha^{k l}(y):=\sum_{i, j=1}^{n} a^{i j} \frac{\partial y_{k}}{\partial x_{i}} \frac{\partial y_{l}}{\partial x_{j}}$ and $\beta^{l}:=\sum_{i=1}^{n} b^{i} \frac{\partial y_{l}}{\partial x_{i}}+\sum_{i, j=1}^{n} a^{i j} \frac{\partial^{2} y_{l}}{\partial x_{i} \partial x_{j}}$.

Hence by the assumption (2.2) we have

$$
\begin{aligned}
\beta^{n}(O) & =\left(\sum_{i=1}^{n} b^{i} \frac{\partial y_{n}}{\partial x_{i}}+\sum_{i, j=1}^{n} a^{i j} \frac{\partial^{2} y_{n}}{\partial x_{i} \partial x_{j}}\right)(P) \\
& =\left(-\sum_{i=1}^{n-1} b^{i} \frac{\partial f}{\partial x_{i}}+b^{n}-\sum_{i, j=1}^{n-1} a^{i j} \frac{\partial^{2} f}{\partial x_{i} \partial x_{j}}\right)(P) \\
& >0 .
\end{aligned}
$$

Now we introduce an auxiliary function $h$ of the form

$$
h(y)=-\sum_{i=1}^{n} y_{i}^{2}+\mu y_{n},
$$

where $\mu$ is a positive constant yet to be determined. A direct calculation gives

$$
L h(O)=-2 \sum_{i=1}^{n} \alpha^{i i}(O)+\mu \beta^{n}(O)
$$

and

$$
\frac{\partial h}{\partial y_{n}}(O)=\mu>0
$$

Note that we have $\beta^{n}(O)>0$. Hence $\mu$ may be chosen large enough so that $\operatorname{Lh}(O)>0$.

Let us pick up a small rectangle $D_{1}:=\left(-d_{1}, d_{1}\right) \times\left(-d_{2}, d_{2}\right) \times \ldots \times(0, c) \subset V$, with $O \in \partial D_{1}$ and $L h>0$ throughout the domain $D_{1}$. Corresponding to the function $h$ there is an open set $B=\left\{y \in \mathbb{R}^{n}: h(y)>0\right\}$. One sees from the construction of $h$ that $K:=D_{1} \cap B$ is nonempty, and the boundary of $K$ can be divided into two parts: $K_{1}:=\partial K \cap \partial D_{1}, K_{2}:=\partial K \cap \partial B$, with $O \in K_{2}$. Now since $u-u(O)>0$ on $K_{1}$, there is a constant $\epsilon>0$ for which the function $w:=(u-u(O))-\epsilon h \geq 0$ on $K_{1}$. This inequality is also satisfied on $K_{2}$, where $h=0$. Thus we have $L w=L u-c u(O)-\epsilon L h \leq-\epsilon L h<0$ in $K$, and $w \geq 0$ on $\partial K$. The weak minimum principle (see the first paragraph of the proof of Theorem 3.1 in [5]; it is here we need the matrix $\left(a^{i j}\right)$, hence $\left(\alpha^{k l}\right)$, to be semi-positive definite) 
now implies that $w \geq 0$ in $K$. Taking the interior normal derivative at the point $O$, where $w(O)=0$, we have $\frac{\partial w}{\partial y_{n}}(O) \geq 0$, which implies that

$$
\frac{\partial u}{\partial y_{n}}(O) \geq \epsilon \frac{\partial h}{\partial y_{n}}(O)=\epsilon \mu>0 .
$$

Therefore we get, in the original $x$-coordinates,

$$
\begin{aligned}
\frac{\partial u}{\partial \nu}(P) & =\left(1+\sum_{i=1}^{n-1}\left(\frac{\partial f}{\partial x_{i}}\right)^{2}\right) \frac{\partial u}{\partial y_{n}}(O)-\sum_{i=1}^{n-1} \frac{\partial u}{\partial y_{i}}(O) \frac{\partial f}{\partial x_{i}}(P) \\
& >0,
\end{aligned}
$$

as required, since $\frac{\partial u}{\partial y_{i}}(O)=0$ for $i=1, \cdots, n-1$ should hold due to the fact that $u$ attains a minimum at $O$.

The case that $u$ attains a strict maximum at $P \in \partial D$ can be proved by applying the above proved result to $-u$. For $c \leq 0$, the proof is similar.

Remark 2.2. As pointed out in [5] (p. 34), (2.4) can be replaced by

$$
\liminf _{x \rightarrow P} \frac{|u(x)-u(P)|}{|x-P|}>0(<0)
$$

where the angle between the vector $x-P$ and the inner normal vector of $\partial D$ at $P$ is less than $\pi / 2-\delta$ for some fixed positive number $\delta$. We require $u \in C^{1}(\bar{D})$ in essence just to obtain that the tangential derivatives of $u$ along $\partial D$ are zero at $P$, which is used to derive (2.13).

2.2. Proof of Theorem 1.1. We first prove part (i) of Theorem 1.1.

Suppose $\varphi$ is a $C^{2}$ solution to problem (1.1)-(1.4). Then by (1.1), it is straightforward to check that $\psi=\varphi_{b}-\varphi$ satisfies

$$
\sum_{i, j=1}^{2} a^{i j}\left(\varphi_{b}\right) \partial_{i j} \psi+b^{1} \partial_{1} \psi+b^{2} \partial_{2} \psi=0,
$$

where

$$
\begin{aligned}
& a^{11}\left(\varphi_{b}\right)=n(x)^{2}\left(c_{b}^{2}-\left(\partial_{1} \varphi_{b}\right)^{2}\right), \\
& a^{12}\left(\varphi_{b}\right)=a^{21}\left(\varphi_{b}\right)=-\partial_{1} \varphi_{b} \partial_{2} \varphi_{b}=0, \\
& a^{22}\left(\varphi_{b}\right)=c_{b}^{2}-\frac{1}{n(x)^{2}}\left(\partial_{2} \varphi_{b}\right)^{2}=c_{b}^{2},
\end{aligned}
$$

with $c_{b}=\sqrt{c_{0}-\frac{\gamma-1}{2}\left(\partial_{1} \varphi_{b}\right)^{2}}$ the sonic speed corresponding to $\varphi_{b}$, and

$$
\begin{aligned}
b^{1}= & -\left(\frac{\gamma+1}{2} n(x)^{2} \partial_{11} \varphi+\frac{\gamma-1}{2} \partial_{22} \varphi\right) \partial_{1}\left(\varphi+\varphi_{b}\right) \\
& +n(x) n^{\prime}(x)\left(c_{b}^{2}+\frac{\gamma-1}{2} \partial_{1} \varphi \partial_{1}\left(\varphi+\varphi_{b}\right)\right), \\
b^{2}= & \frac{\gamma-1}{2} \partial_{2} \varphi \partial_{11} \varphi+2 \partial_{1} \varphi \partial_{12} \varphi+\frac{\gamma+1}{2} \frac{1}{n(x)^{2}} \partial_{2} \varphi \partial_{22} \varphi \\
& +\frac{\gamma-3}{2} \frac{n^{\prime}(x)}{n(x)} \partial_{1} \varphi \partial_{2} \varphi .
\end{aligned}
$$

Recall that $\varphi_{b}$ is a subsonic-sonic flow, so equation (2.14) is a linear degenerate elliptic equation of $\psi$ : it is strictly elliptic in $\mathcal{M} \cup \Sigma_{0}$ and degenerate on $\Sigma_{1}$. 
The boundary conditions of $\psi$ are

$$
\begin{array}{ll}
l_{0} \cdot D \psi=0 & \text { on } \quad \Sigma_{0}, \\
l_{1} \cdot D \psi=0 & \text { on } \quad \Sigma_{1},
\end{array}
$$

where $D \psi=\left(\partial_{1} \psi, \partial_{2} \psi\right)$ and

$$
l_{0}=\left(\partial_{1}\left(\varphi+\varphi_{b}\right), \partial_{2} \varphi / n(0)^{2}\right), \quad l_{1}=\left(\partial_{1}\left(\varphi+\varphi_{b}\right), \partial_{2} \varphi\right) .
$$

By $\left(H_{2}\right)$ and the fact that $\partial_{1} \varphi_{b}>0,(2.20)$ and (2.21) are both oblique derivative conditions.

Now we apply maximum principles to the problems (2.14), (2.20) and (2.21). If $\psi$ is not a constant, by the strong maximum principle, which is valid in any compact subset of $\mathcal{M}$, the maximum and minimum of $\psi$ can only be achieved on $\Sigma_{0}$ or $\Sigma_{1}$. By the classical Hopf boundary point lemma (Lemma 3.4 in [5]), it is only possible to achieve the extremum on $\Sigma_{1}$, i.e., the boundary where the equation is degenerate.

To show that $\psi$ is a constant, by Theorem 2.1 and an argument of contradiction, we just need to show the validity of (2.2). In our situation, it is exactly

$$
b^{1}<0 \quad \text { on } \Sigma_{1} .
$$

The calculation is straightforward and we omit it (cf. (2.8) and also note that $y_{n}=-(x-1)$ now $)$.

Since $\partial_{1} \varphi_{b}>0, \partial_{1} \varphi \geq 0$ and $n^{\prime}(1)=0, n(1)=1$ by $\left(H_{1}\right)\left(H_{2}\right)$, (2.22) is guaranteed by

$$
\frac{\gamma+1}{2} \partial_{11} \varphi+\frac{\gamma-1}{2} \partial_{22} \varphi>0 \quad \text { on } \Sigma_{1} .
$$

We show this is true under the assumption (1.10).

In fact, by the equation (1.1), we obtain that on $\Sigma_{1}$,

$$
c^{2} \Delta \varphi=\left(\partial_{1} \varphi\right)^{2} \partial_{11} \varphi+2 \partial_{1} \varphi \partial_{2} \varphi \partial_{12} \varphi+\left(\partial_{2} \varphi\right)^{2} \partial_{22} \varphi .
$$

Differentiating the boundary condition (1.4) with respect to $y$ and multiplying by $\partial_{2} \varphi$, it follows that

$$
\partial_{1} \varphi \partial_{2} \varphi \partial_{12} \varphi+\left(\partial_{2} \varphi\right)^{2} \partial_{22} \varphi=0 .
$$

Substituting this in (2.24) and noting that $c^{2}=\left(\partial_{1} \varphi\right)^{2}+\left(\partial_{2} \varphi\right)^{2}$, then

$$
\left(c^{2}+\left(\partial_{2} \varphi\right)^{2}\right) \Delta \varphi=c^{2} \partial_{11} \varphi>0 .
$$

So $\Delta \varphi>0$ on $\Sigma_{1}$ and (2.23) follows. (Note that $c=b_{1}>0$ on $\Sigma_{1}$.) This finishes the proof of part (i) of Theorem 1.1 .

We continue to prove part (ii) of Theorem 1.1. The only difference is the boundary condition (2.20). Now it should be written as

$$
l_{0} \cdot D \psi=b_{0}^{2}-B(y) \quad \text { on } \Sigma_{0} .
$$

We consider the case $B \geq b_{0}^{2}$. Suppose $\psi$ is not a constant. Then by the classical Hopf lemma, the minimum of $\psi$ can only be achieved on $\Sigma_{1}$. But this is contradictory to (2.22) and Theorem 2.1. So $\psi$ must be a constant. Hence on $\Sigma_{0}, b_{0}^{2} \equiv B(y)$ should hold. However, we have assumed that $B$ is not identical to $b_{0}^{2}$, so the only 
possibility is that there is no $C^{2}$ solution of the problems (1.1), (1.2), (1.11) and (1.4). The case $B \leq b_{0}^{2}$ can be proved similarly by considering the maximum of $\psi$.

This finishes the proof of Theorem 1.1

Remark 2.3. We see that the equation (2.14) is reduced to

$$
c_{b}^{2} \partial_{22} \psi+b^{1} \partial_{1} \psi+b^{2} \partial_{2} \psi=0
$$

on $\Sigma_{1}$. So (2.22) implies that this equation is similar to a heat equation. This type of degeneracy also occurs, for example, in shock reflection phenomena (cf. [2]).

\section{ACKNOWLEDGMENT}

The authors sincerely thank the referee for providing many valuable comments and suggestions.

\section{REFERENCES}

1. G.-Q. Chen, C. M. Dafermos, M. Slemrod and D. Wang, On two-dimensional sonic-subsonic flow, Comm. Math. Phys. 271 (2007), 635-647. MR2291790 (2008e:35149)

2. G.-Q. Chen and M. Feldman, Global solutions to shock reflection by large-angle wedges for potential flow, Ann. of Math., to appear.

3. G.-Q. Chen and H. Yuan, Uniqueness of transonic shock solutions in a duct for steady potential flow, J. Differential Equations 247 (2009), 564-573. MR2523692

4. R. Courant and K. O. Friedrichs, Supersonic Flow and Shock Waves, Interscience Publishers, Inc., New York, 1948. MR0029615 (10:637c)

5. D. Gilbarg and N. S. Trudinger, Elliptic Partial Differential Equations of Second Order, second edition, Springer, Berlin-New York, 1983. MR737190 (86c:35035)

6. Q. Han and J.-X. Hong, Isometric Embedding of Riemannian Manifolds in Euclidean Spaces, Mathematical Surveys and Monographs, 130, American Mathematical Society, Providence, RI, 2006. MR2261749 (2008e:53055)

7. E. H. Kim, Subsonic solutions for compressible transonic potential flows, J. Differential Equations 233 (2007), 276-290. MR2290280 (2008c:35095)

8. E. H. Kim, Subsonic solutions to compressible transonic potential problems for isothermal selfsimilar flows and steady flows, J. Differential Equations 233 (2007), 601-621. MR2292520 $(2008 \mathrm{c}: 35180)$

9. A. G. Kuz'min, Boundary-Value Problems for Transonic Flow, John Wiley \& Sons, West Sussex, 2002.

10. L. Liu and H. Yuan, Stability of cylindrical transonic shocks for the two-dimensional steady compressible Euler system, J. Hyperbolic Differ. Equ. 5 (2008), 347-379. MR2420002 (2009j:35273)

11. L. Liu and H. Yuan, Global uniqueness of transonic shocks in divergent nozzles for steady potential flows, SIAM J. Math. Anal. 41 (2009), 1816-1824.

12. C. S. Morawetz, Mixed equations and transonic flow, J. Hyperbolic Differ. Equ. 1 (2004), 1-25. MR2052469 (2005f:35259)

13. O. A. Oleĭnik and E. V. Radkevič, Second Order Equations with Nonnegative Characteristic Form, Translated from the Russian by Paul C. Fife, Plenum Press, New York-London, 1973. MR0457908 (56:16112)

14. L. M. Sibner and R. J. Sibner, Transonic flow on an axially symmetric torus, J. Math. Anal. Appl. 72 (1979), 362-382. MR.552343 (80k:58004)

15. P. Šolín and K. Segeth, Non-uniqueness of almost unidirectional inviscid compressible flow, Appl. Math. 49 (2004), 247-268. MR2059429 (2005f:76083)

16. K. Taira, Diffusion Processes and Partial Differential Equations, Elsevier, Singapore, 2004. MR0954835 (90m:60089)

17. H. Yuan, Examples of steady subsonic flows in a convergent-divergent approximate nozzle, J. Differential Equations 244 (2008), 1675-1691. MR2404435 (2009b:35276) 
18. H. Yuan and Y. He, Transonic potential flows in a convergent-divergent approximate nozzle, J. Math. Anal. Appl. 353 (2009), 614-626. MR2508963

19. Y. Zheng, Existence of solutions to the transonic pressure-gradient equations of the compressible Euler equations in elliptic regions, Comm. Partial Differential Equations 22 (1997), 1849-1868. MR1629498 (99c:35191)

Department of Mathematics, East China Normal University, Shanghai 200241, People's Republic of China

E-mail address: pliu@math.ecnu.edu.cn

Department of Mathematics, East China Normal University, Shanghai 200241, People's Republic of China

E-mail address: hryuan@math.ecnu.edu.cn, hairongyuan0110@gmail.com 